\title{
Chronic Cutaneous Disorders Are Associated with a More Favorable Disease Presentation and Outcome in Patients with Hodgkin Lymphoma
}

\author{
Katja Marin ${ }^{a, b}$ Päivi Auvinen ${ }^{a, b}$ Hanne Kuitunenc ${ }^{c}$ Outi Kuittinen $^{a, b}$ \\ ${ }^{a}$ Centre of Oncology, Kuopio University Hospital, Kuopio, Finland; ${ }^{b}$ Oncology, Institute of Clinical Medicine, \\ School of Medicine, Faculty of Health Medicine, University of Eastern Finland (UEF), Kuopio, Finland; ' ${ }^{2}$ epartment of \\ Oncology and Radiotherapy, Oulu University Hospital, Oulu, Finland
}

\section{Keywords}

Hodgkin lymphoma · Chronic inflammation · Cutaneous/ dermatological diseases - Treatment outcome

\begin{abstract}
Introduction: In epidemiological studies, chronic inflammation or previous major infection have revealed to be associated with an increased risk of developing Hodgkin lymphoma $(\mathrm{HL})$. The association of chronic inflammation with the disease outcome is poorly defined. In this retrospective study based on 92 consecutive HL patients, we explored the incidence of previous inflammatory processes or previous major infection in newly diagnosed $\mathrm{HL}$ patients and their association with treatment outcome. Methods: Medical history before lymphoma diagnosis including previous infection; dental inflammation; cutaneous problems; and inflammatory respiratory, gastrointestinal, or musculoskeletal diseases was collected from the patient records. Also clinical $\mathrm{HL}$ presentation, given treatments, and the disease outcome were recorded. Results: Forty-six percent of $\mathrm{HL}$ patients had some of the studied inflammatory factor at the time of diagnosis. Chronic dermatological diseases were present in $16.3 \%$ of patients, and they were associated with an improved relapse-free survival ( $p=$ 0.028 ). Dermatological issues were also associated with earlystage disease and the absence of B-symptoms. Other studied inflammatory factors were not associated with any clinical variables or treatment outcome. Conclusion: Our results demonstrated that among patients with $\mathrm{HL}$, preexisting cutaneous symptoms are associated with a limited-stage disease, the absence of B-symptoms, and favorable prognosis.
\end{abstract}

(c) 2022 The Author(s).

Published by S. Karger AG, Basel

\section{Introduction}

Hodgkin lymphoma (HL), a malignant lymphoproliferative disorder of B-cell origin, is one of the most common cancers in adolescents and young adults. In Finland, 169 new cases were diagnosed in 2014, demonstrating a trend to minor increase in incidence (Finnish Cancer registry). Treatment decisions in HL are based on the disease stage and risk factors. In limited-stage disease, clinical risk factors include number of affected nodal sites, Bsymptoms, and the presence of a bulky tumor. Among patients with an advanced-stage disease, the International Prognostic Score (IPS) is used for prognostic evaluation [1]. Treatment consists of polychemotherapy with or without radiotherapy. Most patients with limited-stage disease are cured from their lymphoma, and even in advanced stages, over $80 \%$ of patients achieve long-term remission with standard therapies $[2,3]$.

Risk factors for developing a HL are not fully discovered. About one-third of patients have a detectable Epstein-Barr virus genome in the malignant cells [4]. Hereditary factors also play a role; classical HL (cHL) in a parent or a sibling increases the disease risk 6-8-fold [5, 6], at least in young adulthood. Third, a well-established, etiological factor is immunosuppression, for example, HIV infection [7].

Our physicians in the Kuopio University Hospital (KUH) had made a clinical observation that many of our HL patients presented with some kind of chronic cutaneous disorder or other infectious or inflammatory condition prior to diagnosis. Inflammatory conditions consisted, for example, from untreated denture, widespread 
Table 1. Patient demographics and the disease outcome in patients with cutaneous problems

\begin{tabular}{|c|c|c|c|c|c|}
\hline & \multicolumn{2}{|l|}{ All patients } & \multicolumn{2}{|c|}{$\begin{array}{l}\text { Patients with cutaneous } \\
\text { problems }\end{array}$} & \multirow[t]{2}{*}{$p$ value } \\
\hline & $n=92$ & $\%$ & $n=15$ & $\%$ & \\
\hline \multicolumn{6}{|l|}{ Age, years } \\
\hline Median & 31 & & & & \\
\hline Range & $16-83$ & & & & \\
\hline Age $<60$ versus $>61$ & 76 versus 16 & 82.6 versus 17.4 & 15 versus 0 & 100 versus 0 & 0.052 \\
\hline \multicolumn{6}{|l|}{ Sex } \\
\hline Male & 49 & 53.3 & 9 & 60 & \multirow{2}{*}{0.567} \\
\hline Female & 43 & 46.7 & 6 & 40 & \\
\hline \multicolumn{6}{|l|}{ Histology } \\
\hline $\mathrm{cHL}$ & 80 & 87 & 12 & 80 & \multirow{2}{*}{0.382} \\
\hline NLPHL & 12 & 13 & 3 & 20 & \\
\hline \multicolumn{6}{|l|}{ Stage $\mathrm{e}^{\mathrm{a}}$} \\
\hline Early stage & 44 & 47.8 & 12 & 80 & \multirow{4}{*}{0.006} \\
\hline Early favorable & 20 & 45 & 4 & & \\
\hline Early unfavorable & 24 & 55 & 8 & & \\
\hline Advanced & 48 & 52.2 & 3 & 20 & \\
\hline \multicolumn{6}{|l|}{ IPS } \\
\hline IPS 0-2 & 33 & 68.8 & 3 & 100 & \multirow{2}{*}{0.228} \\
\hline IPS 3-7 & 15 & 31.3 & 0 & 0 & \\
\hline B-symptoms present & 33 & 35.9 & 1 & 6.7 & 0.01 \\
\hline Relapse & 21 & 22.8 & 0 & 0 & 0.028 \\
\hline Early stage & 3 & 14.3 & & & \\
\hline Advanced & 18 & 85.7 & & & \\
\hline $\mathrm{CHL}$ & 20 & 95.2 & & & \\
\hline NLPHL & 1 & 4.8 & & & \\
\hline Death & 24 & 26.1 & 1 & 6.7 & 0.061 \\
\hline $\mathrm{cHL}$ & 22 & 91.7 & 1 & 100 & \\
\hline NLPHL & 2 & 8.3 & 0 & 0 & $0 . / 58$ \\
\hline \multicolumn{6}{|l|}{ Primary treatment } \\
\hline ABVD & 75 & 81.5 & & & \\
\hline $\mathrm{CHOP}, \mathrm{COEP}$, and R-CHOP & 8 & 8.7 & & & \\
\hline BEACOPP and Dexa-BEAM & 4 & 4.3 & & & \\
\hline Rituximab & 1 & 1.1 & & & \\
\hline No treatment or radiation only & 4 & 4.3 & & & \\
\hline
\end{tabular}

cHL, classical Hodgkin lymphoma; NLPHL, nodular lymphocyte predominant Hodgkin lymphoma; IPS, International Prognostic Score. ${ }^{a}$ According to German Hodgkin Study Group classification.

acne or dermatitis or severe pharyngeal infection. For this reason, we decided to perform a retrospective analysis exploring, which proportion of our patients really had a history of chronic inflammation or inflammatory process before HL diagnosis and whether it had some influence on clinical disease presentation or the outcome in HL.

\section{Material and Methods}

This is a retrospective study based on 92 consecutive HL patients treated in the KUH between 2000 and 2015. Medical history including previous diseases was collected, paying special attention to inflammatory processes like dental inflammation; cutaneous problems; and inflammatory respiratory, gastrointestinal, or musculoskeletal diseases. Also, we checked if there had been any major infection several years or months before lymphoma diagnosis, ex- cluding fever and other general symptoms occurring during last 3 months before diagnosis. Clinical stage according to German Hodgkin Study Group definitions and risk factors [8], given treatment, and the outcome were recorded. Data were collected from the electronic database available at KUH. The study was approved by the Ethics Committee of the North-Savo Health Care District.

The status of the teeth was classified as good (no need for treatment) or poor (patient had untreated teeth and needed immediately consultation of the dentist). Cutaneous problem was positive, if patient had a history of long-term (several months or years) dermatological diagnosis; acne, dermatitis, erythema nodosum, or psoriasis. Pruritus, which is a frequent paraneoplastic symptom in hematological malignancies and HL [9], was not classified as a cutaneous problem. Inflammatory respiratory disease included patients with diagnosed asthma. Colitis ulcerosa, Crohn disease, celiac disease, prolonged chronic gastric inflammation, or diverticulitis was regarded as gastrointestinal diseases. Inflammatory musculoskeletal disease included only rheumatoid arthritis; patients with arthrosis or other joint pain syndromes were excluded. History of major infec- 
Table 2. Incidence of studied inflammatory factors

\begin{tabular}{lll}
\hline Factor & $N$ & $\%$ \\
\hline Cutaneous problems & 15 & 16.3 \\
Poor denture & 9 & 9.8 \\
Previous infection & 16 & 17.4 \\
Gl inflammation & 7 & 7.6 \\
Musculoskeletal inflammation & 1 & 1.1 \\
Respiratory & 4 & 4.3 \\
No inflammatory factors & 50 & 54 \\
\hline
\end{tabular}

tion was positive if there had been a severe infection (hospitalization or antimicrobial medication needed) at least 3 months before lymphoma diagnosis, therefore excluding fever and other nonspecific infectious symptoms typically present at the time of diagnosis of HL.

The statistical analyses were done using SPSS-X software for Windows. The associations between inflammatory history and clinical parameters were calculated with the $\chi^{2}$ test with two-sided $p$ values of less than 0.05 considered to indicate statistical significance. The overall survival (OS) of the patients was calculated with the Kaplan-Meier method. Potential confounding factors were analyzed with multivariate analysis (Cox test), including histological diagnosis, clinical stage, the presence of B-symptoms, IPS, and age. The data cutoff date was January 31,2019 , to analyze relapse-free survival (based on the time from initial diagnosis to progression at any time or relapse) and OS (based on the time from initial diag nosis to death from any cause). For the statistical analysis, the studied factors were classified as positive or negative.

\section{Results}

\section{Patient Population}

This study included 92 patients. Patient demographics is presented in Table 1. The median age of the patients was 31 years (range 16-83 years), and the median followup time was 7.6 years (range $0.3-18.6$ years). There were 44 patients diagnosed with early-stage disease and 48 patients with advanced-stage disease. Eighty patients had cHL, and 12 patients had nodular lymphocyte predominant HL (NLPHL).

\section{Disease Outcome}

Three patients with early-stage disease and 18 patients in the advanced-stage group relapsed. Among patients with cHL, 20 relapses were detected, and the mean time from diagnosis to relapse was 1.5 years (range 0.16-4.9 years). Only 1 patient with NLPHL has relapsed. In advanced stages, the IPS correlated with the risk of relapse since all the patients with 4 or more IPS points relapsed. Twenty-four patients have died, and HL was classified as a cause of death in 10 patients.

\section{Prevalence of Study Parameters}

Incidences of studied factors are shown in Table 2. Altogether, 42 patients had some of the studied inflamma- tory factor at the time of diagnosis, and 9 of them had maximum of 2 risk factors simultaneously. Chronic cutaneous problems were found in 15 patients, and those were more common among patients with early-stage than in patients with advanced-stage disease $(p=0.006)$ (Table 1). Nine patients had dental problems, and 4 patients had asthma at the time of diagnosis. Previous severe infection was detectable in 16 patients. Those were typically respiratory infections (pharyngeal infection, bronchitis, pneumonia, and mononucleosis), but also three central nervous infections (encephalitis and meningitis) were detected. Although pruritus was not classified as a cutaneous problem, it was recognized in 12 of our patients.

\section{Correlation of Study Parameters with the Clinical \\ Outcome}

Chronic dermatological diseases were associated with a favorable outcome (Fig. 1a-d) in the whole study population and also in patients with cHL. Relapse-free survival was significantly better among all patients having cutaneous problems $(p=0.028)$ and also in cHL patients $(p=$ $0.04)$. In OS, there was a trend to a better outcome ( $p=$ 0.071 ) for whole study population. As previously mentioned, dermatological issues were also associated with early-stage disease, and patients were also less likely to have B-symptoms before diagnosis $(p=0.01)$. In multivariate analysis, cutaneous disorders lost their independent predictive value. Dental problems; respiratory, gastrointestinal, and musculoskeletal diseases; or previous infection were not associated statistically significantly with the risk of relapse, death, or clinical variables (cHL vs. NLPHL, stage, and B-symptoms).

\section{Discussion and Conclusions}

In the present study, we found, for the first time, that preexisting cutaneous symptoms are associated with limited-stage disease, the absence of B-symptoms, and favorable prognosis among patients with HL. Cutaneous manifestation of HL is extremely rare, but there are some case reports considering nonspecific cutaneous symptoms in HL. They are reported to occur in $15-50 \%$ of cases [10, $11]$. The effect of these cutaneous symptoms to the treatment outcome has not been studied previously.

Inflammation is an established risk factor for developing cancer, and conversely, malignant cells seem to induce inflammatory microenvironment promoting tumor survival, proliferation, angiogenesis, and metastasis [12]. Previous epidemiological studies have revealed a strong connection between chronic inflammation and all subtypes of cHL in patients with a history of autoimmune disorders and inflammatory conditions (rheumatoid ar- 

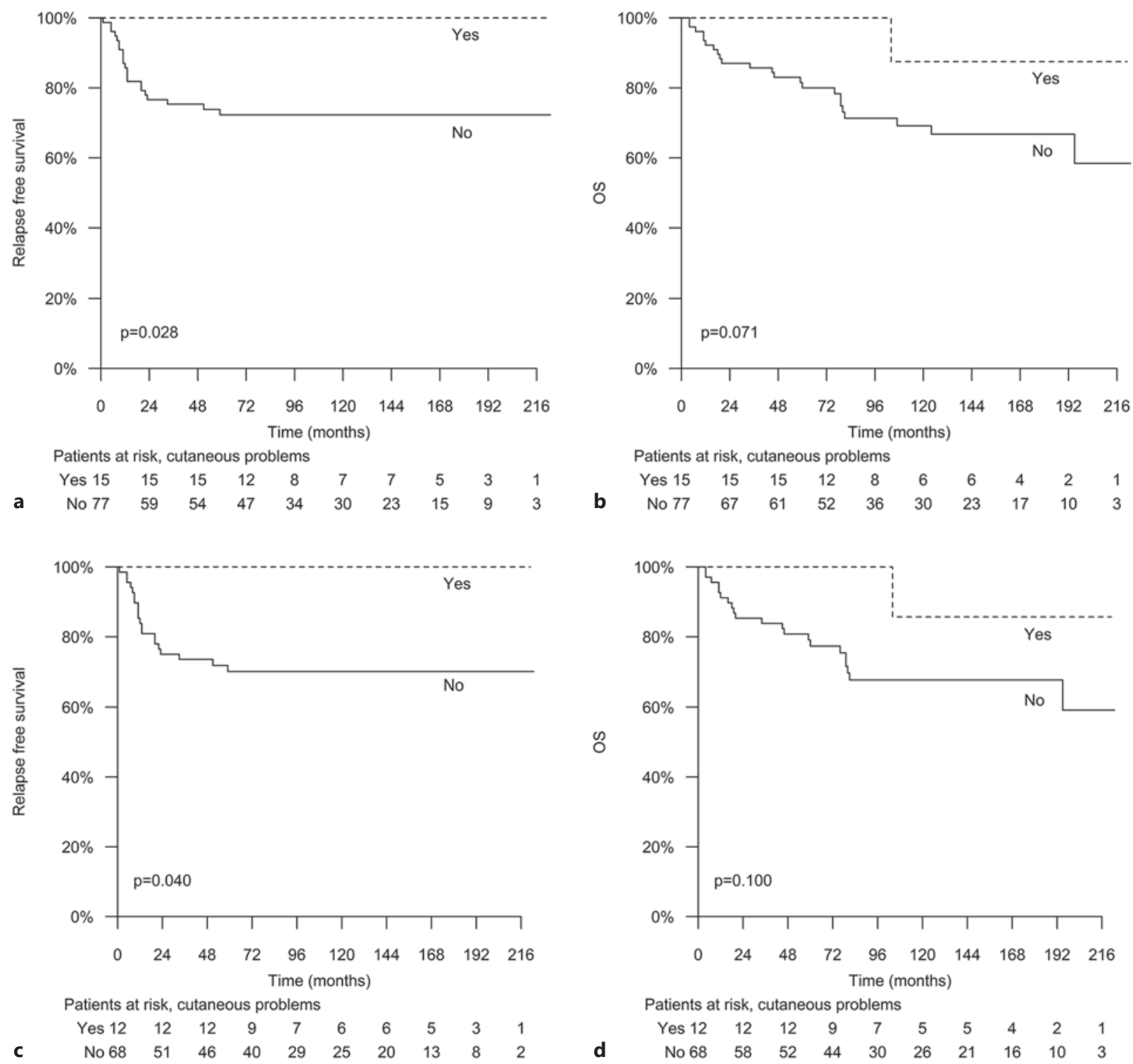

Fig. 1. Relapse-free survival and OS of all HL patients (a, b) and patients with classical HL (c, d) having chronic cutaneous problems.

thritis, systemic lupus erythematosus, sarcoidosis and immune thrombocytopenic purpura, autoimmune hemolytic anemia, polyarteritis nodosa, and psoriasis), especially among older age-groups $[13,14]$. In pediatric patients, children with HL were more likely to have an infection during the previous year before the diagnosis of $\mathrm{HL}$ when compared to healthy controls [15]. Also, in a casecontrol study in the UK, adolescents and adults had significantly more visits to their general practitioners for infections beginning 10 years prior to HL diagnosis [16]. A large Swedish case-control study found significant correlation between any previous infection and risk of HL, but chronic inflammation was not associated with increased lymphoma risk in this study [17]. Recently, a large follow-up study found an association between periodontal disease and the risk of developing non-Hodgkin lymphoma [18]. This is an example of increasing knowledge about immune dysregulation and inflammation beyond lymphomagenesis. Effective treatment of periodontal disease may serve as a primary prophylaxis to malignancy but also to many systemic diseases, among others cardiovascular diseases and diabetes [19-21]. However, the association of these factors with the disease outcome is poorly defined. 
Altogether, $46 \%$ of our patients had some kind of inflammatory process at the time of diagnosis. This number seems high, but unfortunately, in our study, we do not have a control group. Moreover, even if we would be able to verify an increased incidence of these issues, in a retrospective setting, it is not possible to draw any conclusions considering their causality. It is possible that these conditions themselves predispose the patient for developing a lymphoma. Another explanation might be that they present only a sign of the same preexisting immunological problem that predispose the patient developing HL. However, our study clearly points out that most of these inflammatory conditions do not interfere with the disease outcome. Cutaneous and/or dental problems were discovered in $25 \%$ of our patients, which is a quite a low proportion when compared to studies made in Finnish population. In large birth cohort studies, inflammatory cutaneous diseases were present in about a quarter of study population [22], and the incidence of severe dental problems was found to be as high as $25-35 \%$ of the study population [23]. While other inflammatory conditions did not correlate with the risk of relapse, it seemed that chronic cutaneous disease was associated with a favorable outcome, and actually none of these patients suffered a disease relapse. These patients had also a more limited-stage disease and less often B-symptoms. No differences regarding sex or other clinical variables associating to cutaneous disorders was detected, except there was a trend toward these patients to be younger ( $<60$ years, $p=0.052$ ). Prognosis of young patients with HL is generally much better than in older patients [24], which may be a confounding factor in our study. In multivariate analysis, $\mathrm{cu}-$ taneous issues lost their independent significance. Because this is a retrospective study, we are not able to make definitive conclusions regarding the causality of these associations. We speculate that there might be two explanations for our finding. One explanation could be that a HL provoked by a chronic cutaneous disorder is biologically more benign and responsive to therapy. Other hypothesis might be that lymphoma is diagnosed more promptly among patients having regular contact with a physician treating their cutaneous disease. However, in our patients, the first undisputed symptom of HL was always detected by a patient himself as a prominent lymph node.

This is the first time that the association of chronic cutaneous problems with the favorable clinical disease presentation and prognosis is described among patients with HL. Our retrospective study material is quite small but includes all HL cases diagnosed in our area during a period of 15 years. The small number of cases makes it difficult to draw definitive conclusions between studied inflammatory factors and treatment outcome of HL, and this should be regarded as a hypothesis generation study, which should be verified in larger materials. It would also be interesting to go deeper to the disease biology and find out whether HL in patients with cutaneous issues differs biologically from other HL cases or whether this association reflects common etiology between these two conditions.

\section{Acknowledgments}

The authors acknowledge the biostatistician Tuomas Selander for graphical expertise.

\section{Statement of Ethics}

The study was approved by the Ethics Committee of the NorthSavo Health Care District (ref. 375/2015). According to Finnish legislation, no informed consent from the study population is required for this retrospective, noninterventional study.

\section{Conflict of Interest Statement}

The authors have no conflicts of interest to declare.

\section{Funding Sources}

There are no funding sources to declare.

\section{Author Contributions}

K.M.: data collection and analysis and manuscript writing. P.A.: study design and manuscript writing. H.K.: manuscript writing and editing. O.K.: data analysis and manuscript writing. All of the authors revised this paper critically and approved the version for publication.

\section{Data Availability Statement}

The dataset used and analyzed during the current study are available from the corresponding author upon reasonable request.

\section{References}


4 Murray P, Bell A. Contribution of the Epstein-Barr Virus to the pathogenesis of Hodgkin lymphoma. Curr Top Microbiol Immunol. 2015;390(Pt 1):287-313.

5 Crump C, Sundquist K, Sieh W, Winkleby MA, Sundquist J. Perinatal and family risk factors for Hodgkin lymphoma in childhood through young adulthood. Am J Epidemiol. 2012 Dec;176(12):1147-58

6 Kharazmi E, Fallah M, Pukkala E, Olsen JH, Tryggvadottir L, Sundquist K, et al. Risk of familial classical Hodgkin lymphoma by relationship, histology, age, and sex: a joint study from five Nordic countries. Blood. 2015 Oct; 126(17):1990-5.

7 Grulich AE, Vajdic CM. The epidemiology of cancers in human immunodeficiency virus infection and after organ transplantation. Semin Oncol. 2015 Apr;42(2):247-57.

8 German Hodgkin Study Group 2022. The GHSG's risk group classification system. Available from: https://en.ghsg.org/diseasestages (accessed January 6, 2022).

9 Weisshaar E, Weiss M, Mettang T, Yosipovitch G, Zylicz Z; Special Interest Group of the International Forum on the Study of Itch. Paraneoplastic itch: an expert position statement from the Special Interest Group (SIG) of the International Forum on the Study of Itch (IFSI). Acta Derm Venereol. 2015 Mar;95(3): 261-5.

10 Vrotsos E, Dosal J, Zaiac M, Alexis J. Pityriasis rosea-like cutaneous eruption as the presenting symptom of Hodgkin lymphoma. Case report and review of the literature. J Dermatol Case Rep. 2015 Sep;9(3):81-4.
11 Hayes TG, Rabin VR, Rosen T, Zubler MA. Hodgkin's disease presenting in the skin: case report and review of the literature. J Am Acad Dermatol. 1990 May;22(5 Pt 2):944-7.

12 Mantovani A, Allavena P, Sica A, Balkwill F. Cancer-related inflammation. Nature. 2008 Jul;454(7203):436-44.

13 Hollander P, Rostgaard K, Smedby KE, Chang ET, Amini RM, de Nully Brown P, et al. Autoimmune and atopic disorders and risk of classical Hodgkin lymphoma. Am J Epidemiol. 2015 Oct;182(7):624-32.

14 Fallah M, Liu X, Ji J, Försti A, Sundquist K, Hemminki K. Hodgkin lymphoma after autoimmune diseases by age at diagnosis and histological subtype. Ann Oncol. 2014 Jul;25(7): 1397-404.

15 Linabery AM, Erhardt EB, Fonstad RK, Ambinder RF, Bunin GR, Ross JA, et al. Infectious, autoimmune and allergic diseases and risk of Hodgkin lymphoma in children and adolescents: a Children's Oncology Group study. Int J Cancer. 2014 Sep;135(6):1454-69.

16 Newton R, Crouch S, Ansell P, Simpson J, Willett EV, Smith A, et al. Hodgkin's lymphoma and infection: findings from a UK casecontrol study. Br J Cancer. 2007 Nov;97(9): 1310-4.

17 Kristinsson SY, Gao Y, Bjorkholm M, Lund $\mathrm{SH}$, Sjoberg J, Caporaso N, et al. Hodgkin lymphoma risk following infectious and chronic inflammatory diseases: a large population-based case-control study from Sweden. Int J Hematol. 2015 Jun;101(6):563-8.
18 Bertrand KA, Shingala J, Evens A, Birmann BM, Giovannucci E, Michaud DS. Periodontal disease and risk of non-Hodgkin lympho$\mathrm{ma}$ in the health professionals follow-up study. Int J Cancer. 2017 Mar;140(5):1020-6.

19 Munoz Aguilera E, Suvan J, Buti J, Czesnikiewicz-Guzik M, Barbosa Ribeiro A, Orlandi M, et al. Periodontitis is associated with hypertension: a systematic review and meta-analysis. Cardiovasc Res. 2020 Jan;116(1):28-39.

20 Liccardo D, Cannavo A, Spagnuolo G, Ferrara N, Cittadini A, Rengo C, et al. Periodontal disease: a risk factor for diabetes and cardiovascular disease. Int J Mol Sci. 2019 Mar;20(6): 1414.

21 Bui FQ, Almeida-da-Silva CLC, Huynh B, Trinh A, Liu J, Woodward J, et al. Association between periodontal pathogens and systemic disease. Biomed J. 2019 Feb;42(1):27-35.

22 Sinikumpu SP, Huilaja L, Jokelainen J, Koiranen M, Auvinen J, Hägg PM, et al. High prevalence of skin diseases and need for treatment in a middle-aged population. A Northern Finland Birth Cohort 1966 study. PLoS One. 2014 Jun 9;9(6):e99533.

23 Harju P, Hausen H, Huumonen S, Knuuttila M, Könönen M, Nordblad A, et al. Oral health in the Finnish adult population: Health 2000 Survey. 2008. Available from: https://urn.fi/ URN: ISBN: 978-951-740-852-3 (accessed January 6, 2022).

24 Borchmann S, Engert A, Böll B. Hodgkin lymphoma in elderly patients. Curr Opin Oncol. 2018 Sep;30(5):308-16. 